\title{
Une expérience de mixité dans l'enseignement secondaire à la fin des années 1930 : le lycée Marcelin Berthelot à Saint-Maur-des-Fossés
}

\section{Cécile Hochard}

\section{CpenEdition}

Journals

Édition électronique

URL : https://journals.openedition.org/clio/614

DOI : $10.4000 /$ clio.614

ISSN : 1777-5299

Éditeur

Belin

Édition imprimée

Date de publication : 1 novembre 2003

Pagination : 113-124

ISBN : 2-85816-706-0

ISSN : 1252-7017

Référence électronique

Cécile Hochard, «Une expérience de mixité dans l'enseignement secondaire à la fin des années 1930 le lycée Marcelin Berthelot à Saint-Maur-des-Fossés », Clio. Histoire, femmes et sociétés [En ligne], 18। 2003, mis en ligne le 04 décembre 2006, consulté le 23 avril 2022. URL : http:// journals.openedition.org/clio/614; DOI : https://doi.org/10.4000/clio.614

Ce document a été généré automatiquement le 23 avril 2022

Tous droits réservés 


\title{
Une expérience de mixité dans l'enseignement secondaire à la fin des années 1930 : le lycée Marcelin Berthelot à Saint-Maur-des-Fossés
}

\author{
Cécile Hochard
}

1 Une stricte séparation des sexes domine le système scolaire public français, et singulièrement l'enseignement secondaire, jusqu'aux années 1960. Cette séparation puise sa justification dans la définition d'identités et de « destins » féminin et masculin fondamentalement différents, ce qui ne permet pas d'envisager la réunion des élèves dans des établissements mixtes. Pourtant, en octobre 1938, le lycée de garçons Marcelin Berthelot, à Saint-Maur-des-Fossés, accueille des filles dès son ouverture. À la charnière des années trente et quarante, la situation est exceptionnelle. L'événement est aussi singulier que peu connu.

2 Pourquoi une telle situation, en principe impossible, a-t-elle pu voir le jour? L'étude exige de restituer l'ensemble des facteurs qui l'ont rendue possible, de multiplier les niveaux d'analyse - évolution des identités de sexe, mais aussi contexte politique, composition sociale des groupes en présence, poids des circonstances locales. Par sa singularité même, l'expérience menée à Marcelin Berthelot révèle, au-delà des formes les plus visibles du fonctionnement du champ scolaire, toute la complexité des niveaux de réalité qui ont pu conduire, pour une part, aux transformations des années soixante. Un lycée mixte en 1938 : un objet scolaire non identifié ?

L'existence d'un lycée mixte n'est, a priori, pas concevable dans l'enseignement public français de la fin des années 1930. Sur le plan institutionnel comme sur le plan des représentations, le système scolaire - et en particulier l'enseignement secondaire - est marqué, depuis le début du XIX ${ }^{e}$ siècle, par son caractère d'abord exclusivement masculin. Le droit à l'instruction, progressivement accordé aux filles, s'accompagne d'une stricte séparation des sexes que ne viennent pas démentir quelques très rares exceptions, telles les écoles de hameaux à classe unique où la mixité est officiellement 
tolérée depuis 1884. L'accession des filles à l'enseignement secondaire, reconnue par la loi Camille Sée en 1880, ne peut se faire que dans des établissements spécifiques. Jamais la question d'un possible regroupement des élèves dans des établissements communs n'est alors évoquée, tant les buts assignés à l'enseignement secondaire diffèrent selon le sexe des élèves ${ }^{1}$. Les lycées et collèges de filles ne sont pas censés préparer leurs élèves au Baccalauréat ni à une accession à la sphère professionnelle, mais bien les former à leur rôle futur de mère et d'épouse tout en les soustrayant à l'emprise de l'Église. Le concept même de "mixité", tel que nous le comprenons aujourd'hui, n'existe pas dans les premières décennies du $\mathrm{XX}^{\mathrm{e}}$ siècle ${ }^{2}$.

4 Si la question du regroupement des élèves dans un même établissement commence à faire débat, au début du siècle, en particulier parmi les pédagogues qui s'appuient sur des exemples étrangers, notamment anglo-saxons, on parle alors presque exclusivement de «coéducation». Ces débats, qui prolongent les réflexions sur les «destins » masculin et féminin, sur les formes et les finalités de l'enseignement dispensé, restent toutefois peu importants à l'intérieur du champ scolaire et ne donnent lieu à aucune application ${ }^{3}$. La stricte séparation des sexes perdure pendant les trois premières décennies du secondaire féminin et les transformations de la demande de scolarisation féminine - en particulier du droit à passer le Baccalauréat - qui aboutissent, en 1924, à l'assimilation des contenus des enseignements masculin et féminin, ne remettent pas en cause le principe des établissements distincts.

Depuis le milieu des années 1910, et surtout au cours des années 1920, des instructions officielles du ministère de l'Instruction publique laissent apparaître quelques entorses au principe de la séparation des sexes. La première de ces entorses concerne les " petites classes » des lycées et collèges. Une circulaire ministérielle du 23 novembre 1915 autorise «l'entrée des petites filles dans les classes primaires des établissements de garçons, et réciproquement $»^{4}$. Cette mesure sera étendue, neuf ans plus tard, aux classes élémentaires (c'est-à-dire la Huitième et la Septième). La deuxième entorse est liée au processus d'assimilation et intéresse les classes terminales des établissements. $\mathrm{Au}$ début des années 1920, le nombre de cours «clandestins» de préparation au Baccalauréat se multiplie dans les lycées féminins, dont les élèves sont de plus en plus nombreuses à se présenter à l'examen. En octobre 1922, le ministre de l'Instruction publique, Léon Bérard, prend officiellement acte de cette multiplication et, alors même que les cursus féminin et masculin sont, dans les textes, encore différenciés, décide que " provisoirement et sans créer un précédent pour les autres classes, les jeunes filles, en Mathématiques et en Philosophie exclusivement, quand leur nombre n'est pas suffisant pour justifier la création de cours particuliers, pourront être admises à suivre les cours de l'établissement de garçons $»^{5}$. Les motifs invoqués pour justifier cette mesure visent à obtenir un triple résultat : satisfaire les familles, réaliser une importante économie, tenter un essai pédagogique. L'admission de filles dans les établissements de garçons est étendue à d'autres sections au cours de l'année 1923 : tout d'abord aux classes préparatoires des Grandes Écoles auxquelles les filles ont accès, puis à la classe de Première, sous réserve toujours que cette section n'existe pas dans l'établissement féminin du lieu de résidence. Il est extrêmement difficile d'évaluer le nombre d'établissements qui ont pu être affectés par ces différentes mesures et plus encore celui des filles effectivement scolarisées parmi des garçons. Tout juste peut-on noter que, dans les lycées parisiens, au cours des années 1930, les demandes d'inscription dans les classes préparatoires aux Grandes Écoles concernent chaque année plus d'une trentaine d'élèves. Encore faut-il bien voir que, dans l'esprit des autorités, il ne s'agit là 
en aucune manière d'une étape vers la " mixité » puisque, dès que les lycées féminins disposent des sections terminales et préparatoires aux Grandes Écoles, les filles sont censées y retourner. L'assimilation doit apporter paradoxalement un renforcement de la séparation des enseignements.

6 Toutes ces instructions qui, dans le quotidien des établissements, produisent des situations ponctuelles de mélange des sexes, sont strictement limitées et ne concernent jamais le noyau dur des études secondaires : les classes de Sixième à Seconde. De ce fait, l'entrée de 25 filles à Marcelin Berthelot, dans les sections de premier et second cycles, en octobre 1938, puis le rapide accroissement de leur nombre, constitue bien un événement exceptionnel.

Pourquoi des filles à Marcelin Berthelot, lycée de garçons?

$7 \mathrm{Au}$ cours de la première année de fonctionnement du lycée Marcelin Berthelot, établissement situé dans le département de la Seine, à une quinzaine de kilomètres de la capitale, 25 filles sont donc présentes parmi 525 garçons. Un an plus tard, on en compte 94, pour 605 élèves masculins. Le pourcentage de filles s'accroît fortement et ne cesse d'augmenter à partir de la rentrée d'octobre 1940 pour atteindre, en 1945, $39 \%$, avec 515 filles pour 805 garçons $^{6}$. Comment rendre compte d'une telle présence qui, compte tenu des règles de fonctionnement du système scolaire, n'aurait jamais dû être possible?

8 L'arrivée des filles à Marcelin Berthelot ne résulte ni d'une évolution globale des représentations collectives en matière de différence de sexe, encore moins d'un mouvement imprimé d'en haut, par les autorités universitaires. Il faut souligner, d'emblée, le poids des facteurs circonstanciels, au premier rang desquels la période troublée de la guerre et du régime de Vichy. Qu'une telle période ait un effet favorable peut sembler paradoxal, puisque ces années sont celles de la remise au goût du jour "l'éternel féminin ${ }^{7}$; cependant, la désorganisation des services centraux, la gestion pragmatique et souvent locale des établissements favorisent, le cas échéant, des situations imprévues. Encore n'y a-t-il là qu'un facteur permissif.

9 L'impulsion vient des parents d'élèves. C'est la demande de scolarisation qui constitue le facteur fondamental. Le rôle et l'action des parents sont essentiels, tout comme ils l'avaient été, dans les années 1920, pour permettre à leurs filles d'accéder aux classes terminales des lycées de garçons. On trouve par exemple, datée de septembre 1938, une demande d'inscription rédigée par un professeur de l'école primaire supérieure de Saint-Maur, qui souhaite voir ses deux filles poursuivre leurs études dans le nouveau lycée. Les arguments avancés par cet enseignant sont uniquement d'ordre pratique: gain de temps, de santé, d'argent et de sécurité pour ses enfants et lui-même, leur domicile se situant à 5 minutes de Marcelin Berthelot ${ }^{8}$. Le proviseur du lycée accueille cette demande favorablement "étant donné les faibles effectifs qui composeront nos classes moyennes et supérieures pendant les premières années $»^{9}$; en revanche, le recteur exprime ses réticences faisant valoir que «au moment où on sépare les sexes dans les classes d'École, il me paraît difficile de faire un lycée mixte à Saint-Maur. On ouvrirait la voie à toutes sortes de demandes $»^{10}$. Pourtant, un mois plus tard, on l'a vu, le lycée ouvre ses portes et accueille en son sein, avec l'autorisation du ministère, 25 filles ${ }^{11}$. Leur arrivée en masse dans les années suivantes résulte d'un effet d'engrenage : l'administration se trouve prise au piège d'une situation qu'elle ne maîtrise pas. Désireuse de disposer d'un effectif suffisant pour le démarrage du lycée, elle ne peut interdire ensuite aux jeunes filles déjà inscrites d'y poursuivre leur scolarité, alors 
même qu'elle ne parvient pas à tarir les flux d'entrée. L'arrivée des filles est ainsi facilitée par l'impuissance administrative ; son moteur premier n'en reste pas moins la demande des parents d'élèves.

On peut approfondir l'analyse de cette demande de scolarisation en s'intéressant aux origines sociales des élèves concernés ${ }^{12}$. On remarque tout d'abord que le recrutement global du lycée Marcelin Berthelot s'apparente bien davantage à celui d'une école primaire supérieure qu'à celui des «grands " lycées parisiens ${ }^{13}$. Si l'on y retrouve des enfants issus des fractions de classes sociales qui, traditionnellement, fréquentent le lycée - fonctionnaires, moyenne bourgeoisie, professions libérales - on remarque une forte proportion d'enfants de petits employés, de petits commerçants, d'artisans et d'ouvriers, inconnue dans les établissements de la capitale. Ces dernières catégories représentent plus du quart du recrutement de Marcelin Berthelot entre 1940 et 1944, et le tiers entre 1944 et 1948. La comparaison des recrutements féminin et masculin offre des différences sensibles : le recrutement féminin apparaît globalement plus modeste que celui des garçons et les différences sont plus nettes encore pendant les années de guerre $^{14}$. L'examen de l'origine scolaire des élèves vient renforcer cette constatation : une majorité absolue de filles est en effet issue de l'enseignement primaire alors que, du côté des garçons (et compte non tenu des élèves en classe préparatoire vétérinaire), la proportion n'atteint pas les $45 \%$ et que plus du tiers des élèves masculins poursuit, à Marcelin Berthelot, un parcours scolaire commencé dans le secondaire. Dès l'ouverture du lycée, des parents appartenant aux catégories les plus modestes semblent avoir moins de réticences à faire scolariser leurs filles dans un établissement de garçons signe d'un affaiblissement des normes en matière de différences des sexes ? - alors que, dans un premier temps, les classes plus aisées restent en retrait, puis emboîtent le pas un peu plus tard. Faut-il voir, dans l'ouverture d'un lycée nouveau, sans tradition scolaire préétablie, l'opportunité, pour certaines familles, de faire commencer des études secondaires à leurs filles, sans avoir à franchir la barrière symbolique d'un lycée féminin, où la sélection sociale est plus forte?

11 Une analyse en terme "d'offre de place ${ }^{15}$ confirme l'idée d'une accession à l'enseignement secondaire d'élèves-filles qui ne l'auraient sans doute pas fréquenté sans l'ouverture de Marcelin Berthelot. Il faut prendre en compte, pour expliquer les demandes, la quasi-absence, dans cette banlieue sud-est de Paris, d'établissement d'enseignement secondaire de filles. Il existe bien, à Vincennes, un cours secondaire, survivance des établissements créés par Victor Duruy en 1853, mais celui-ci n'a pas le prestige d'un lycée. Les seuls autres établissements qui assurent une scolarité au-delà de l'obligation légale sont des cours complémentaires annexés aux écoles primaires, ainsi qu'une école primaire supérieure, à Saint-Maur, mais elle ne prépare qu'au Brevet supérieur et non au Baccalauréat. Ces établissements sont, de plus, statutairement, rattachés à l'enseignement primaire. Les familles désirant scolariser leur fille dans l'enseignement secondaire doivent donc les inscrire au lycée du Cours de Vincennes (dans le $\mathrm{XX}^{\mathrm{e}}$ arrondissement parisien) ou à Victor Hugo (dans le IIIe), ce qui implique de longs et coûteux déplacements, ces établissements ne comportant pas d'internat. La création d'un lycée à Saint-Maur permet à la fois à des élèves déjà scolarisées dans le secondaire de poursuivre leurs études près de chez elles et, surtout, créée une offre dans laquelle vont bientôt se glisser des centaines d'élèves qui, sans cette opportunité, seraient probablement restées dans une filière d'enseignement primaire. La composition des effectifs d'un établissement est ainsi souvent la résultante non de la 
simple demande, mais de la structure de l'offre proposée. Le lycée Marcelin Berthelot ne semble pas démentir ce renversement des perspectives habituelles.

Une expérience tolérée dans les faits, jamais acceptée dans son principe

12 L'arrivée de filles au lycée Marcelin Berthelot s'est effectuée en quelque sorte par effraction. Analyser l'expérience de "mixité" alors menée pose de nombreux problèmes de méthode et d'interprétation, en particulier si on l'interroge à partir de la question des identités de sexe. Les sources habituellement utilisées en histoire des représentations, au premier rang desquelles la littérature grise, ne permettent pas d'accéder aux racines du processus qui a conduit à cette expérience, pas davantage à l'expérience elle-même. Les sources appropriées, elles, font globalement défaut. Du côté des élèves, aucun témoignage contemporain des faits n'a été retrouvé. Les quelques commentaires existants ont été recueillis il y a quelques années et posent les problèmes inhérents à de tels documents: phénomène de reconstruction de la mémoire, enjolivement des souvenirs, etc. Beaucoup d'anciens élèves évoquent la présence des filles, preuve sans doute de la nouveauté qu'elle constituait alors pour eux; tous se rejoignent pour vanter les mérites de cette "expérience [qui] allait devenir très rapidement bénéfique : incitation, émulation, compétition et vie scolaire plus joyeuse $\aleph^{16}$. Les propos restent convenus et les faits réinterprétés à partir de l'évidence actuelle de la mixité. Qu'en est-il de l'expérience vécue à ce moment-là ? La question reste posée. Les rares sources contemporaines des faits émanent des parents d'élèves et de l'administration du lycée. À peine y trouve-t-on quelques bribes factuelles relatives à la présence des filles : certains enseignants sont mal à l'aise devant ce nouveau public, il n'y aurait finalement que très peu "d'incidents ", la situation multiplie les occasions de contacts entre garçons et filles (discussions, sorties, flirts même). Quant à savoir ce qu'une telle expérience peut produire d'effets sur l'habitus de jeunes gens nés dans l'entre-deux-guerres et issus des classes moyennes urbaines, à peu près rien ne permet de l'étudier.

13 Les rares sources disponibles permettent cependant de se faire une idée du sens que les contemporains ont pu prêter à la situation. Le discours de l'Association des parents d'élèves, qui a joué un rôle déterminant pour obtenir l'admission des filles au lycée, se limite à des considérations strictement pédagogiques et insiste sur les bénéfices apportés par l'expérience, en particulier sur l'émulation née entre élèves des deux sexes. Tout juste quelques pères de familles évoquent, individuellement, les inconvénients de la coéducation, mais sans plus de détail. Les questions morales sont très peu abordées. Elles ne sont pas totalement absentes des propos tenus, mais la réalité de la vie lycéenne aurait rapidement balayé « les inconvénients que l'on pouvait craindre $\aleph^{17}$. En fait l'essentiel semble ailleurs : la question première est celle des usages du système scolaire et rejoint par là même les motifs qui ont suscité un fort accroissement de la scolarisation des filles depuis une vingtaine d'années. L'important pour les familles, en effet, est que leurs filles fréquentent un lycée. Les commentaires favorables sont ceux de parents d'élèves trouvant leur intérêt à la présence d'un tel établissement. Ils révèlent le décalage entre la situation de l'enseignement secondaire des années 1930-1940 et les demandes qui lui sont faites: le caractère mixte du lycée étant relativement de peu importance par rapport à l'existence même de cet établissement.

Du côté de l'administration, la situation présente ne cesse d'apparaître comme un état de fait provisoire, voué à disparaître à terme, la réunion des sexes dans un même lieu 
étant par essence négative. Selon les circonstances, plusieurs séries d'arguments sont utilisées. Des arguments gestionnaires: les filles prennent la place des garçons, «l'encombrement de certaines sections par des jeunes filles» contraignant l'administration «à refuser l'accès du lycée à d'assez nombreux garçons $»^{18}$. Des arguments d'ordre pédagogique : les classes mixtes ne procureraient aucun avantage réel pour l'instruction des élèves ou alors des effets négatifs - «le caractère mixte du lycée complique beaucoup l'œuvre d'éducation ", explique l'un des proviseurs dans un rapport - « le langage qui convient aux garçons n'est pas toujours celui qu'il faut tenir aux jeunes filles. (...) Il arrive aussi que les jeunes filles prennent aux garçons leurs défauts et n'observent pas toujours toute la réserve souhaitable, leur présence est un élément de trouble et ne contribue à améliorer ni l'instruction, ni l'éducation ${ }^{19}$. Si l'on considère les discours tenus, on retrouve sans surprise catégories, questions et plus généralement toute une rhétorique issue de la littérature grise: on interroge les différences de «nature» entre filles et garçons, leurs "qualités» respectives présumées et la crainte des situations immorales ne cesse d'apparaître. Encore ceci n'épuise-t-il pas le sens de ces discours. Les efforts constants de l'administration pour limiter les contingents de filles présentes dans l'établissement, mais aussi son obligation de composer avec un état de fait qu'elle ne peut modifier, donnent à ses propos un sens plus complexe. Le souci premier du proviseur est d'assurer la bonne marche de son lycée, d'éviter tout ce qui pourrait en entraver le fonctionnement normal. Si son attention se porte sur les éventuelles perturbations suscitées par la présence féminine, ou encore sur les risques que cette présence fait courir à la réputation du lycée, en même temps, il ne peut qu'entériner la situation de fait de "mixité ", ce qui aboutit d'une certaine façon à la normaliser, alors même que le principe de la réunion des sexes n'est jamais accepté.

$\mathrm{Au}$ total, l'expérience menée à Marcelin Berthelot s'inscrit tout entière à l'intérieur d'un rapport de force dans lequel l'administration désapprouve, mais ne peut modifier la situation. La volonté de limiter la présence féminine dans l'établissement, à défaut de pouvoir la supprimer entièrement, demeure le souci constant des autorités universitaires dont l'action reste marquée par le refus du modèle de coéducation. La présence des filles n'est pourtant jamais enrayée, leurs familles continuant à les inscrire dans l'établissement et, ce, jusqu'à l'instauration officielle de la mixité à Marcelin Berthelot, au début des années 1960.

L'existence d'un lycée mixte, à la fin des années 1930, est bien un événement singulier dans l'histoire du système scolaire public français. L'examen des variables expliquant la présence féminine à Marcelin Berthelot a montré l'importance des facteurs circonstanciels. Une situation de fait s'installe - un établissement mixte fonctionne que ni les réticences des autorités universitaires, ni leurs tentatives pour diminuer la proportion de filles ne parviennent à remettre en cause. S'il faut se garder de chercher là les prémisses trop directes d'un phénomène qui adviendra à la génération suivante, dans un tout autre contexte économique et culturel, le lycée Marcelin Berthelot offre pourtant l'exemple paradoxal d'une situation de mélange des sexes qui, par le poids de la demande parentale, notamment par l'affaiblissement des normes en matière de différences des sexes qu'elle manifeste, préfigure la mixité à venir. 


\section{BIBLIOGRAPHIE}

À Saint-Maur-des-Fossés, en passant par le lycée Marcelin-Berthelot de 1938 à 1988, 1994, Association des anciens élèves du lycée Marcelin-Berthelot.

Larousse du XXe siècle, 6 volumes, 1928-1933.

Pénélope. Pour l'histoire des femmes, 1980, «Éducation des filles. Enseignement des femmes », $n^{\circ} 2$.

Traité sur la coéducation : ses avantages, ses inconvénients, conclusions, 1908, Châtellerault, Imprimerie J. Dagnaud.

BREUSE Édouard, 1970, La coéducation dans les écoles mixtes, Paris, PUF.

BUISSON Ferdinand, 1911, Nouveau dictionnaire de pédagogie et d'instruction primaire, Paris, Hachette.

BURNESS G.-F., 1912, La coéducation dans les écoles secondaires, thèse de doctorat de l'Université de Lille, Imprimerie-Librairie Camille Robbe.

BRIAND Jean-Pierre, CHAPOULIE Jean-Michel, 1981, « L'enseignement primaire supérieur de garçons en France, 1918-1942 », Actes de la recherche en sciences sociales, n 39, p. 87-111.

BRIAND Jean-Pierre, CHAPOULIE Jean-Michel, PÉRETZ Henri, 1979, « Les conditions institutionnelles de la scolarisation des garçons entre 1920 et 1940 », Revue d'histoire moderne et contemporaine, XXVI, p. 391-421.

HOCHARD Cécile, 2002, Les lycées de Paris et de la région parisienne de 1938 à 1947, thèse de doctorat d'histoire contemporaine, université Paris 7-Denis Diderot.

HOUEL Annick, ZANCARINI-FOURNEL Michelle, 2001, École et mixités, Cahiers Masculin/Féminin, Lyon, Presses Universitaires de Lyon.

HUGUENIN Élisabeth, 1929, La coéducation des sexes, expériences et réflexions, Neuchâtel/Paris, Delachaux et Niestlé.

LELIÈVRE Françoise, LELIÈVRE Claude, 1991, Histoire de la scolarisation des filles, Paris, Nathan.

MAYEUR Françoise, 1977, L'Enseignement Secondaire des Jeunes Filles sous la Troisième République, Paris, Presses de la Fondation Nationale des Sciences Politiques.

MOSCONI Nicole, 1989, La mixité dans l'enseignement secondaire, un faux-semblant ?, Paris, PUF.

MUEL-DREYFUS Francine, 1996, Vichy et l'éternel féminin. Contribution à une sociologie politique de l'ordre des corps, Paris, Le Seuil.

PARDÉ Marcelle, 1939, « La coéducation des sexes », in Célestin Bouglé (dir.), Encyclopédie française, tome XV, IIIe partie.

\section{NOTES}

1.Sur cette question, on consultera Mayeur 1977 et Lelièvre 1991.

2.Si l'adjectif « mixte » est employé depuis le XIXe siècle, il désigne aussi bien la coexistence du privé et du public que la réunion, dans une même classe, d'élèves de 
sections ou de sexes différents, sans que ce dernier sens prédomine. On peut noter que le mot « mixité » ne figure pas dans le Larousse du XXe siècle, édité entre 1928 et 1933.

3.On se reportera notamment à Buisson 1911, Burness 1912, Huguenin 1929 ou encore à Pardé 1939.

4.AN, AJ/16/8679, Le ministre de l'Instruction publique et des Beaux-Arts à MM. les recteurs (Paris, le 23 novembre 1915).

5.AN, AJ/16/8679, Le ministre de l'Instruction publique et des Beaux-Arts à MM. les recteurs (Paris, le 23 octobre 1922).

6.De plus, si on examine uniquement les effectifs des classes des premier et second cycles, on arrive à $42 \%$ de filles dans les classes secondaires, le lycée Marcelin Berthelot comprenant à partir de 1942 un fort contingent d'élèves en classe préparatoire aux écoles vétérinaires (près de $10 \%$ de l'effectif total en 1943 par exemple).

7.Muel-Dreyfus 1996.

8.AN, AJ/16/8572, M. Héroguez à M. le proviseur du lycée Marcelin Berthelot (Murols, le 7 septembre 1938).

9.AN, AJ/16/8572, Le proviseur du lycée Marcelin Berthelot à M. l'inspecteur d'Académie de Paris (Saint-Maur, le 8 septembre 1938).

10.AN, AJ/16/8572, note manuscrite du recteur de l'Académie de Paris.

11.AD Val-de-Marne, Archives du lycée Marcelin Berthelot, 1T/150, Chronique du lycée Marcelin Berthelot (sd, mais 1945).

12. Ces analyses ont pu être menées à bien grâce aux fiches individuelles d'inscription des élèves du lycée, conservées aux archives départementales du Val-de-Marne sous les cotes $1861 \mathrm{~W} / 163$ à 172 . Plus de 660 fiches ont été saisies représentant un effectif cumulé de près de 1800 élèves présents au lycée entre 1938 et 1948, soit un peu plus de $10 \%$ de l'effectif total réel. La validité statistique de cet échantillon a été confirmée grâce à la comparaison des pourcentages de filles et de garçons. Les écarts entre les pourcentages des effectifs réels du lycée et ceux de l'échantillon sont compris entre 1 et $5 \%$ pour les années postérieures à 1940 . Pour les deux premières années de fonctionnement de l'établissement, le faible nombre d'élèves ne permet pas de réaliser des statistiques fiables. Ces fiches renseignent sur l'état civil de chaque élève, son parcours scolaire avant et après son entrée à Marcelin Berthelot, son statut (boursier ou non, externe ou demi-pensionnaire), ainsi que sur la profession du père (ou de la mère quand elle est chef de famille). Les points de comparaison avec les autres lycées de la Seine sont tirés de Hochard, 2002.

13.Le recrutement des EPS a été étudié par Briand, Chapoulie 1981.

14.Si l'on sépare globalement les professions les plus modestes (petits artisans, petits commerçants, comptables, employés, enseignement primaire, ouvriers, contremaîtres, secrétaires) des professions plus élevées (gros artisans, gros commerçants, professeurs de l'enseignement secondaire, haute fonction publique, industriels, ingénieurs, professions libérales, représentants, cadres moyens de la fonction publique), $60 \%$ des filles sont issues de la première catégorie et 38 \% de la seconde entre 1940 et 1944 (les chiffres sont de $48 \%$ et $47 \%$ pour les garçons). Entre 1944 et 1948, les proportions sont de $51 \%$ pour la première catégorie, $47 \%$ pour la seconde $(51 \%$ et $46 \%$ pour les garçons). Les totaux ne sont pas égaux à $100 \%$ car il existe une petite frange de professions diverses, inconnues ou de parents sans profession.

15.Ce modèle est au centre des analyses de Briand, Chapoulie, Péretz 1979.

16.À Saint-Maur-des-Fossés 1994 : 37. 
17.AN, AJ/16/8572, dossier d'établissement : lycée Marcelin Berthelot, le président de l'association des parents d'élèves à M. le recteur (Saint-Maur, le 20 septembre 1940). 18.AN, AJ/16/8572, dossier d'établissement : lycée Marcelin Berthelot, Le proviseur du lycée à M. le recteur de l'Académie de Paris (Saint-Maur-des-Fossés, le 12 mars 1946). 19.AN, AJ/16/8649, Le proviseur du lycée Marcelin Berthelot à M. le recteur de l'Académie de Paris : rapport sur la situation de l'établissement à la fin du premier trimestre 1942-1943 (Saint-Maur, le 6 janvier 1943).

\section{RÉSUMÉS}

La séparation des sexes caractérise le système scolaire français, et tout particulièrement l'enseignement secondaire, jusqu'aux années 1960. Dans la première moitié du XX $\mathrm{XX}^{\mathrm{e}}$ siècle, la mixité, telle que nous l'envisageons aujourd'hui, n'est pas concevable. Pourtant, à sa création, en octobre 1938, le lycée de garçons Marcelin Berthelot, à Saint-Maur-des-Fossés, accueille également des filles. Expliquer cette présence féminine, absolument singulière, oblige à faire la part des facteurs circonstanciels, de l'évolution des identités de sexe et de celle de la demande parentale. Si le lycée continue, en dépit des critiques, à accueillir des élèves des deux sexes jusqu'à l'instauration officielle de la mixité, il ne faut cependant pas voir dans cette expérience une simple anticipation des évolutions postérieures.

Until the 1960's, the French educational system, and especially secondary schools, were dominated by the separation of sexes. In the first half of the twentieth century, coeducational schooling as we know it today was inconceivable. Nonetheless, the Marcelin Berthelot boys' high school in the Paris suburbs accepted girls from its foundation in October 1938. This unusual feminine presence can be explained by a series of mitigating circumstances, the evolution of gendered identities and familial requests. Although the presence of girls was maintained right up to the official instigation of coeducation in this institution, its early existence is not simply an anticipation of later evolutions.

\section{AUTEUR}

\section{CÉCILE HOCHARD}

Cécile HOCHARD est Docteur en histoire contemporaine de l'Université Paris 7-Denis Diderot.

Elle a soutenu, en mars 2002, une thèse sur Les lycées de Paris et de la région parisienne de 1938 à 1947, à paraître chez Belin en 2004. Elle a notamment publié « Des adolescents dans la guerre »(revue Adolescence, 2001) et a présenté au colloque Lycées et lycéens en France 1802-2002, une communication intitulée « Indiscipline et chahuts dans les lycées parisiens (1930-1950) » (à paraître). Elle poursuit actuellement des recherches sur l'enseignement secondaire pendant l'entre-deux-guerres et les années 1940. 